\title{
Cross-complementary subsets in paratopological groups
}

\author{
Weihua Lin ${ }^{1,2}$ (D), Lianhua Fang ${ }^{3}$ (D), Li-Hong Xie ${ }^{* 4}$ (D) \\ ${ }^{1}$ College of Mathematics and Informatics, Fujian Normal University, Fuzhou 350007, P.R. China \\ ${ }^{2}$ School of Mathematics and Statistics Minnan Normal University, Zhangzhou 363000, P.R. China \\ ${ }^{3}$ General Education Center, Quanzhou University of Information Engineering, Quanzhou, 362000, P.R. \\ China \\ ${ }^{4}$ School of Mathematics and Computational Science, Wuyi University, Jiangmen 529020, P.R. China
}

\begin{abstract}
The following general question is considered by A.V. Arhangel'skiǐ [Perfect mappings in topological groups, cross-complementary subsets and quotients, Comment. Math. Univ. Carolin. 2003]. Suppose that $G$ is a topological group, and $F, M$ are subspaces of $G$ such that $G=M F$. Under these general assumptions, how are the properties of $F$ and $M$ related to the properties of $G$ ? Also, A.V. Arhangel'skiǐ and M. Tkachenko [Topological Groups and Related Structures, Atlantis Press, World Sci., 2008] asked what is about the above question in paratopological groups [Open problem 4.6.9, Topological Groups and Related Structures, Atlantis Press, World Sci. 2008]. In this paper, we mainly consider this question and some positive answers to this question are given. In particular, we find many A.V. Arhangel'skiî's results hold for $k$-gentle paratopological groups.
\end{abstract}

Mathematics Subject Classification (2010). 22A05, 54H11, 54D35, 54D60

Keywords. paratopological groups, $k$-gentle paratopological groups, perfect mappings, paracompact $p$-spaces, metrizable groups, countable tightnesses

\section{Introduction}

Recall that a semitopological group is a group with a topology such that the multiplication in the group is separately continuous. A paratopological group is a group with a topology such that the multiplication is jointly continuous. If $G$ is a paratopological group (resp. semitopological group) and the inverse operation of $G$ is continuous, then $G$ is called a topological group (resp. quasitopological group). The reader can find a lot of recent progress about paratopological, semitopological and quasitopological groups in the survey article [11].

In a topological group the product operation generates a variety of continuous mappings. In 2003, A.V. Arhangel'skiir [1] considered the following general questions. Suppose that $G$ is a topological group, $A$ and $B$ are subsets of $G$, and $f$ is the mapping of the product space $A \times B$ onto the subspace $A B$ of $G$ given by the product operation in $G$. Under certain natural restrictions on $A$ and $B$, what can be said about the properties of the

\footnotetext{
*Corresponding Author.

Email addresses: 740816193@qq.com (W. Lin), lotus35@163.com (L. Fang), xielihong2011@aliyun.com, yunli198282@126.com (L.H. Xie)

Received: 22.03.2018; Accepted: 27.08.2018
} 
mapping $f$ ? How the properties of the subspace $A B$ are related to the properties of the subspaces $A$ and $B$ ? In this direction, the next statement was proved in [4, Chapter 3, Section 4, Proposition 1]:

Proposition 1.1. Suppose that $G$ is a topological group, and $F$ a compact subspace of $G$. Then the restriction $f$ of the product mapping $G \times G \rightarrow G$ to the subspace $G \times F$ is a perfect mapping of $G \times F$ onto $G$.

Recall that a continuous mapping $f: X \rightarrow Y$ is perfect if $f$ is a closed mapping such that $f^{-1}(y)$ is compact in $X$ for each $y \in Y$.

Using Proposition 1.1, A.V. Arhangel'skiǐ established some results on above questions as following (see [1] or [3, Chapter 4, section 4.6]):

Theorem 1.2. Suppose that $F$ is a compact subspace of a topological group $G$, and that $M$ a closed subspace of $G$. Suppose also that both $M$ and $F$ have some of the following property:

(1) both $M$ and $F$ have countable tightness (see [1, Theorem 8] or [3, Theorem 4.6.8];

(2) both $M$ and $F$ are metrizable (see [1, Corollary 10] or [3, Theorem 4.6.9];

(3) $M$ is paracompact (see [1, Theorem 14] or [3, Theorem 4.6.11]);

(4) $M$ is a paracompact p-space [1, Theorem 7].

Then both $M F$ and $F M$ are closed with the same property. If in addition, $G=F M$, then $G$ has the same property.

Theorem 1.3. (see [1, Theorem 15] or [3, Theorem 4.6.12]) Suppose that $G$ is a topological group such that $G=F M$, where $F \subseteq G$ is compact and $M$ is a Cech-complete closed subspace of $G$. Then $G$ is a Cech-complete topological group, and both $M$ and $G$ are paracompact.

In 2008, A.V. Arhangel'skiǐ and M. Tkachenko posed the following question:

Question 1.4. [3, Open problem 4.6.9] Which theorems in this section remain true for paratopological groups?

In this paper, we give some positive answers to Question 1.4 for $k$-gentle paratopological groups (Definition see below). In this paper, we mainly prove that: (1) Suppose that $G$ is a paratopological group, and $H$ a metrizable invariant topological subgroup of $G$ such that $H$ has a countably tight compact grasp on $G$; then $G$ is a metrizable topological group, which improves [1, Proposition 1] (see Theorem 2.3); (2) let $G$ be a paratopological group and $H$ a closed invariant subgroup of $G$ such that $H$ has a countably tight compact grasp on $G$ and the pseudocharacter of $H$ is countable; then the pseudocharacter of $G$ is also countable, which improves [1, Theorem 2] (see Theorem 2.5). Also, we show that Theorems 1.2 and 1.3 hold for $k$-gentle paratopological groups (see Theorems 2.14 and $2.15)$

We follow notation and terminology in [3] and [5]. We assume regular separation axiom in this paper.

\section{Main results}

Let $G$ be a paratopological group, and $A$ and $B$ subsets of $G$. Let us say that $A$ and $B$ are cross-complementary (in $G$ ) if $G=A B=\{a b: a, b \in G\}$. Suppose that $\mathcal{P}$ is a topological or algebraic-topological property, and $A$ is a subset of $G$. We will say that $A$ has a $\mathcal{P}$-grasp on $G$ if there exists a subset $B$ of $G$ such that $B$ is cross-complementary to $A$ and has the property $\mathcal{P}$. In particular, $A \subseteq G$ has a compact grasp on $G$ if there exists a compact subspace $B$ of $G$ such that $A B=G$. Similarly, $A$ has a metrizable grasp on $G$ if $G=A B$, for some metrizable subspace $B$ of $G$. 
Firstly, we consider the countably tight compact, countable pseudocharacter-grasp on a paratopological group $G$ for a closed invariant subgroup $H \subseteq G$. Some results in [1] are improved. Recall that a space $X$ is countable tightness if for any subset $A \subseteq X$ and any point $x \in \bar{A}$ there is countable set $B \subseteq A$ such that $x \in \bar{B}$. A space $X$ is countably tight compact which means that $X$ is compact and countable tightness.

Lemma 2.1. [9, Proposition 5.5] Every locally compact paratopological group is a topological group.

Proposition 2.2. [1, Proposition 1] Suppose that $G$ is a topological group, and $H$ a metrizable invariant subgroup of $G$ such that $H$ has a countably tight compact grasp on $G$. Then $G$ is metrizable.

The above result can be strengthened as following:

Theorem 2.3. Suppose that $G$ is a paratopological group, and $H$ a metrizable invariant topological subgroup of $G$ such that $H$ has a countably tight compact grasp on $G$. Then $G$ is a metrizable topological group.

Proof. According to Proposition 2.2, it is enough to show that $G$ is a topological group. Let us consider now the quotient group $G / H$ and the quotient mapping $p: G \rightarrow G / H$. Since there is a compact set $B \subseteq G$ such that $H B=G$, we have $p(B)=G / H$. Thus $G / H$ is a compact paratopological group. By Lemma $2.1 \mathrm{G} / \mathrm{H}$ is a topological group. Note that $H$ is a topological group, so it follows that $G$ is a topological group from [8, Lemma 4].

The proof of [3, Lemma 4.6.6] is valid for Lemma 2.4. For the sake of the completeness, we give its proof.

Lemma 2.4. Let $H$ be a closed subgroup of a paratopological group $G$. If both the group $H$ and the quotient space $G / H$ have countable pseudocharacter, then so has $G$.

Proof. Let $p: G \rightarrow G / H$ be the quotient mapping. Choose a countable family $\gamma$ of open sets in $G / H$ such that $\bigcap \gamma=\{p(e)\}$, where $e$ is the neutral element of $G$. Also, choose a countable family $\beta$ of open sets in $G$ such that $H \cap \cap \beta=\{e\}$. Then the family $\alpha=\beta \cup\left\{p^{-1}(U): U \in \gamma\right\}$ is countable, consists of open sets in $G$, and $\cap \alpha=\{e\}$. Therefore, the pseudocharacter of $G$ at $e$ is countable and, by homogeneity, so is at every point $x \in G$.

Theorem 2.5. Let $G$ be a paratopological group and $H$ a closed invariant subgroup of $G$ such that $H$ has a countably tight compact grasp on $G$ and the pseudocharacter of $H$ is countable. Then the pseudocharacter of $G$ is also countable.

Proof. Let $p: G \rightarrow G / H$ be the quotient mapping. Since $H$ is closed and there is a compact set $B \subseteq G$ with countable tightness such that $H B=G$, we have $p(B)=G / H$. Thus $G / H$ is a compact $T_{1}$ paratopological group. By Lemma $2.1 G / H$ is a $T_{1}$ topological group. Note that $B$ is a compact set with countable tightness and perfect mappings do not increase the tightness. Since $p(B)=G / H$ and $p$ is a perfect mapping, $G / H$ is a compact countable tightness topological group. Thus, by [3, Corollary 4.2.2] $G / H$ is metrizable, then the statement directly follows from Lemma 2.4.

Theorem 2.6. Suppose that $G$ is a normal paratopological group, and let $H$ be a closed subgroup of $G$ such that the pseudocharacter of $H$ is countable and $H$ has a cosmic grasp $^{\circ}$ on $G$. Then the pseudocharacter of $G$ is also countable.

\footnotetext{
${ }^{\dagger}$ A subsets family $\beta$ of a space $X$ is called a network, if for each open set $U$ and each $x \in U$ there is an $F \in \beta$ such that $x \in F \subseteq U$. A space $X$ is cosmic if $X$ has a countable network.
} 
Proof. Let $p: G \rightarrow G / H$ be the quotient mapping. According to Lemma 2.4, it is enough to show that $G / H$ has a countable pseudocharacter. Since $G$ is normal and $H$ is closed, one can easily show that $G / H$ is Hausdorff. Since there is a subset $B \subseteq G$ with a countable network such that $H B=G, p(B)=G / H$ has also a countable network $\alpha$. Put $\beta=\{\bar{a}$ : $a \in \alpha, e \notin \bar{a}\}$. Clearly, $\beta$ is countable. Now we show that $\bigcap\{G / H \backslash b: b \in \beta\}=\{p(e)\}$. By contradiction assume that there is a point $x \in \bigcap\{G / H \backslash b: b \in \beta\}$ such that $x \neq p(e)$. Since $G / H$ is Hausdorff, there is $b \in \alpha$ such that $x \in b$ and $e \notin \bar{b}$. Thus $x \notin G / H \backslash b$. This is a contradiction.

Next, we shall discuss the cross-complementary in a $k$-gentle paratopological group $G$ for a compact set $F \subseteq G$. Recall that a paratopological group $G$ is called $k$-gentle if $F^{-1}$ is compact for each compact set $F \subseteq G$.

Lemma 2.7. Let $F$ be a compact set of a paratopological group $G$. If one of the following conditions

(1) $G$ is a k-gentle paratopological group,

(2) $F$ is a subgroup,

is satisfied, then the mapping $s: F \rightarrow F^{-1}$ defined as $s(x)=x^{-1}$ for each $x \in F$ is a homeomorphism.

Proof. (1) It is obvious that $s$ is a one-to-one mapping. Firstly, we shall prove that $s$ is closed. Take any closed set $A$ in $F$. Then $A$ is compact. Since $G$ is a $k$-gentle paratopological group, $A^{-1}=s(A)$ is compact. Thus $s(A)$ is closed. This proves that $s$ is a closed mapping.

Next, we shall prove that $s$ is continuous. In fact, since $G$ is a $k$-gentle paratopological group and $F$ is compact, $F^{-1}$ is compact. Thus each closed set $B \subseteq F^{-1}$ is compact, so $s^{-1}(B)=B^{-1}$ is compact. And hence $s^{-1}(B)$ is closed. This implies that $s$ is continuous.

(2) Since $F$ is a compact paratopological group, $F$ is a topological group by Lemma 2.1. Thus $s$ is a homeomorphism.

Proposition 2.8. Let $F$ be a compact subspace of a paratopological group $G$. If one of the following conditions

(1) $G$ is a k-gentle paratopological group,

(2) $F$ is a subgroup,

is satisfied, then the restriction $f$ of the product mapping $G \times G \rightarrow G$ to the subspace $F \times G$ is a perfect and open mapping of $F \times G$ onto $G$. The same is valid for the restriction of the product mapping to the subspace $G \times F$ of $G \times G$.

Proof. Define a mapping $s: F \times G \rightarrow F^{-1} \times G$ as $s(x, y)=\left(x^{-1}, x y\right)$, for each $(x, y) \in$ $F \times G$.

Firstly, we shall prove that $s$ is a one-to-one continuous mapping. It is obvious that $s$ is one-to-one. Take any open neighborhood $V \times U$ of $\left(x^{-1}, x y\right)$ in $F^{-1} \times G$. By Lemma 2.7, there is an open neighborhood $W_{1}$ of $x$ such that $W_{1}^{-1} \subseteq V$. Since $G$ is a paratopological group, there are open neighborhoods $O_{1}$ and $O_{2}$ of $x$ and $y$, respectively, in $G$ such that $O_{1} O_{2} \subseteq U$. Put $W=W_{1} \cap O_{1}$. Then one can easily show that $s\left(W \times O_{2}\right) \subseteq V \times U$. This implies that $s$ is continuous.

Similarly, we can easily show that the inverse mapping $s^{-1}: F^{-1} \times G \rightarrow F \times G$ defined as $s^{-1}(x, y)=\left(x^{-1}, x y\right)$ for each $(x, y) \in F \times G$ is continuous. Hence, $s$ is a homeomorphism.

Let $p: F^{-1} \times G \rightarrow G$ be the natural projection mapping given by $p(x, y)=y$ for each $(x, y) \in F^{-1} \times G$. Since $x y=p\left(x^{-1}, x y\right)=p(s(x, y))$ for each $(x, y) \in F \times G$, we conclude that $f$ is the composition of $s$ and $p$, that is, $f=p \circ s$. Note that $F^{-1}$ is compact, so the mapping $p$ is perfect by [5, Theorem 3.1.16]. Therefore, the mapping $f$, being a composition of a homeomorphism with a perfect mapping, is itself perfect. Also, note 
that $p$ is an open mapping and $s$ is a homeomorphism. Thus the mapping $f$, being a composition of $p$ and $s$, is itself open.

Since the mapping $i$ of $G \times G$ onto itself, defined by $i(x, y)=(y, x)$ for $x, y \in G$, is a homeomorphism, and $i(F \times G)=G \times F$, the rest of the proposition is immediate.

Lemma 2.9. Let $F$ be a compact set of a k-gentle paratopological group $G$ and $M$ be a closed set in $G$. Then $F M$ and $M F$ is also closed in $G$.

Proof. We will show that $F M$ is closed in $G$. The case of $M F$ differs only in trivial details. Take any point $a \notin F M$. It follows that the sets $F^{-1} a$ and $M$ are disjoint. Since $G$ is a $k$-gentle paratopological group, $F^{-1} a$ is compact. Therefore, by [3, Theorem 1.4.29], there is an open neighbourhood $U$ of $e$ such that $F^{-1} a U$ and $M$ are disjoint. Therefore, $a U$ and $F M$ are disjoint. Since $a U$ is an open neighbourhood of $a$, we conclude that $a$ is not in closure of $F M$. Hence, $F M$ is closed in $G$.

Corollary 2.10. Suppose that $F$ is a compact subspace of a $k$-gentle paratopological group $G$, and let $M$ be a closed subspace of $G$. Then the restriction $f$ of the product mapping $G \times G \rightarrow G$ to the subspace $M \times F$ is a perfect mapping of $M \times F$ onto a closed subspace of $G$.

Proof. To derive this corollary from Proposition 2.8, we only have to observe that the restriction of a perfect mapping to a closed subspace is again a perfect mapping and that the product of a compact set with a closed set in a $k$-gentle paratopological group is closed by Lemma 2.9 .

The following result is well known.

Lemma 2.11. Let $F$ be a compact subgroup of a paratopological group $G$ and $M$ be a closed set in $G$. Then $F M$ and $M F$ is also closed in $G$.

Applying Proposition 2.8 and Lemma 2.11, one can easily obtain the following result.

Corollary 2.12. Suppose that $F$ is a compact subgroup of a paratopological group $G$, and let $M$ be a closed subspace of $G$. Then the restriction $f$ of the product mapping $G \times G \rightarrow G$ to the subspace $M \times F$ is a perfect mapping of $M \times F$ onto a closed subspace of $G$.

Corollaries 2.10 and 2.12 are the main technical tool in this article. [1, Example 6] shows that the perfect mapping of $M \times F$ onto $M F$ in Corollary 2.10 need not be open, even for topological groups.

Proposition 2.13. Let $F$ be a compact subspace of a paratopological group G. Suppose that either $F$ is a subgroup or $G$ is a $k$-gentle paratopological group, and that $M$ is a closed subspace of $G$. Suppose also that both $M$ and $F$ have a topological property $\mathcal{P}$ such that so has the product space $M \times F$ and that the property $\mathcal{P}$ is preserved by perfect mapping. Then $M F$ has also the property $\mathcal{P}$. If in addition, $G=F M$, then so has $G$.

Proof. Since the product space $M \times F$ has the property $\mathcal{P}$ and $M F$ is the perfect image of $M \times F$ by Corollaries 2.10 and 2.12, according to the assumption $M F$ has also the property $\mathcal{P}$.

The following result shows that Theorem 1.2 holds for $k$-gentle paratopological groups.

Theorem 2.14. Suppose that $F$ is a compact subspace of a $k$-gentle paratopological group $G$, and that $M$ is a closed subspace of $G$. Suppose also that both $M$ and $F$ have some of the following property:

(1) both $M$ and $F$ have countable tightness;

(2) both $M$ and $F$ are metrizable;

(3) $M$ is paracompact; 
(4) $M$ is a paracompact p-space;

(5) $M$ is countablely paracompact

Then both $M F$ and $F M$ have the same property. If in addition, $G=F M$, then $G$ has the same property.

Proof. (1) The tightness of $M \times F$ is countable (see [2]). Perfect mappings do not increase the tightness [2]. Then the statement follows from Proposition 2.13.

(2) $M \times F$ is metrizable and perfect mappings preserve the metrizability. Then the statement follows from Proposition 2.13.

(3) Since the products of paracompact spaces and compact spaces are paracompact and perfect mappings preserve the paracompactness [5], the assertion follows from Proposition 2.13 .

(4) Indeed, $M \times F$ is a paracompact $p$-space and $M F$ is an image of $M \times F$ under a perfect mapping, by Corollary 2.10. Now it follows from a remarkable theorem of V.V. Filippov [6] that $M F$ is a paracompact $p$-space as well.

(5) Since the products of countablely paracompact spaces and compact spaces are countablely paracompact and perfect mappings preserve the countable paracompactness [5], the statement follows from Proposition 2.13.

The next result is somewhat unexpected: it shows that certain closed subsets of a $k$ gentle paratopological group with a compact grasp on this group must be paracompact topological group.

Theorem 2.15. Suppose that $G$ is a k-gentle paratopological group such that $G=F M$, where $F \subseteq G$ is compact and $M$ is a Čech-complete closed subspace of $G$. Then $G$ is a Cech-complete topological group, and both $M$ and $G$ are paracompact.

Proof. The product of Čech-complete space and a compact space is Čech-complete. Therefore, the space $M \times F$ is Čech-complete. Since Cech-completeness is preserved by perfect mappings, $G=F M$ is Cech-complete by Proposition 2.13. Therefore, $G$ is a topological group (see [3]). However, every Cech-complete topological group is paracompact [10]. Hence, $G$ is paracompact. Since $M$ is a closed subspace of $G$, the space $M$ is also paracompact.

Finally, if $F$ is a subgroup in Theorems 2.14 and 2.15, by Proposition 2.13 and Corollary 2.12 , then we can weaken $G$ to be a paratopological group in Theorems 2.14 and 2.15.

Theorem 2.16. Suppose that $F$ is a compact subgroup of a paratopological group $G$, and that $M$ a closed subspace of $G$. Suppose also that both $M$ and $F$ have some of the following property:

(1) both $M$ and $F$ have countable tightness;

(2) both $M$ and $F$ are metrizable;

(3) $M$ is paracompact;

(4) $M$ is paracompact p-space;

(5) $M$ is countablely paracompact

Then both $M F$ and $F M$ have the same property. If in addition, $G=F M$, then $G$ has the same property.

Theorem 2.17. Suppose that $G$ is a paratopological group such that $G=F M$, where $F \subseteq G$ is a compact subgroup and $M$ is a Čech-complete closed subspace of $G$. Then $G$ is a Čech-complete topological group, and both $M$ and $G$ are paracompact.

Remark 2.18. In [7], there is a quasitopological group $G$, which is neither metrizable nor Cech-complete, such that $G=F M$, where $F$ is a compact metrizable subgroup of $G$ and $M$ is a discrete closed subspace in $G$ (see [7, Example 3.1]). Thus the items (1) and (2) of Theorem 2.16 and Theorem 2.17 are not valid for quasitopological groups. 
Acknowledgment. This research is supported by NSFC (Nos. 11601393,11861018). The authors would like to give thanks to the referee for his valuable comments and suggestions for the improvement of this paper.

\section{References}

[1] A.V. Arhangel'skiǐ, Perfect mappings in topological groups, cross-complementary subsets and quotients, Comment. Math. Univ. Carolin. 44, 4, 701-709, 2003.

[2] A.V. Arhangel'skiǐ and V.I. Ponomarev, Fundamentals of General Topology: Problems and Exercises, Reidel, 1984.

[3] A.V. Arhangel'skiǐ and M. Tkachenko, Topological Groups and Related Structures, Atlantis Press, World Sci. 2008.

[4] N. Bourbaki, Elements de Mathematique, Premiere Partie, Livre 3, Ch. 3, 3-me ed., Hermann, Paris, 1949.

[5] R. Engelking, General Topology, Revised and completed edition, Heldermann Verlag, 1989.

[6] V.V. Filippov, On perfect images of paracompact p-spaces, Soviet Math. Dokl. 176, 533-536, 1967.

[7] P. Li and L. Mou, On quasitopological groups, Topology Appl. 161, 243-247, 2014.

[8] O. Ravsky, On H-closed paratopological groups, Visnyk Lviv. Univ. Ser. Mat. Mekh. 59, 96-101, 2001.

[9] O. Ravsky, The topological and algebraic properties of paratopological groups, Ph.D. thesis. Lviv University, 2003 (in Ukrainian).

[10] W. Roelke and S. Dierolf, Uniform Structures on Topological Groups and Their Quotients, McGraw-Hill, New York, 1981.

[11] M. Tkachenko, Paratopological and semitopological groups vs topological groups, in: K.P. Hart, J. van Mill, P. Simon (Eds.), Recent Progress in General Topology III, Springer Atlantis Press, 803-859, 2013. 